Spin Physics (SPIN2014)

International Journal of Modern Physics: Conference Series

Vol. 40 (2016) 1660090 (7 pages)

(C) The Author(s)

DOI: $10.1142 /$ S2010194516600909

\title{
Polarization Preservation and Control in a Figure-8 Ring
}

\author{
Ya. S. Derbenev*, V. S. Morozov*, ${ }^{\Uparrow}$, F. Lin*, Y. Zhang*, A. M. Kondratenko ${ }^{\dagger}$, \\ M. A. Kondratenko ${ }^{\dagger}$ and Yu. N. Filatov ${ }^{\ddagger}, \S$ \\ *Thomas Jefferson National Accelerator Facility, Newport News, Virginia 23606, USA \\ †Science and Technique Laboratory "Zaryad", Novosibirsk 630090, Russia \\ ${ }^{\ddagger}$ Moscow Institute of Physics and Technology, Dolgoprudny 141700, Russia \\ $\S$ Joint Institute for Nuclear Research, Dubna 141980, Russia \\ Imorozov@jlab.org
}

Published 29 February 2016

\begin{abstract}
We present a complete scheme for managing the polarization of ion beams in Jefferson Lab's proposed Medium-energy Electron-Ion Collider (MEIC). It provides preservation of the ion polarization during all stages of beam acceleration and polarization control in the collider's experimental straights. We discuss characteristic features of the spin motion in accelerators with Siberian snakes and in accelerators of figure- 8 shape. We propose $3 \mathrm{D}$ spin rotators for polarization control in the MEIC ion collider ring. We provide polarization calculations in the collider with the 3D rotator for deuteron and proton beams. The main polarization control features of the figure- 8 design are summarized.
\end{abstract}

Keywords: Polarized ion beam; figure-8 ring; 3D spin rotator.

PACS numbers: 29.27.Bd, 29.27.Hj, 41.75.Ak

\section{Introduction}

The main challenge of accelerating polarized ion beams to high energies in synchrotrons with vertical bending field is the necessity to overcome numerous spin depolarizing resonances. Use of solenoidal and helical (dipole) Siberian snakes solves the problem of polarization preservation in the regions of low and high energies, respectively. However, usage of snakes may become problematic in the medium energy range from a few $\mathrm{GeV}$ to a few tens of $\mathrm{GeV}$ due to the longitudinal-field snakes already being too weak while the transverse-field snakes still causing large orbit excursions. Using figure-8 ring geometry is an elegant way to preserve and control the polarization of a beam of any particle species during its acceleration and storage at any energy. ${ }^{1,2}$

This is an Open Access article published by World Scientific Publishing Company. It is distributed under the terms of the Creative Commons Attribution 3.0 (CC-BY) License. Further distribution of this work is permitted, provided the original work is properly cited. 
In a circular collider with two Siberian snakes, the snake spin rotation axes are perpendicular to each other, the spin tune is equal to one half, and only vertical polarization is stable in the collider arcs. ${ }^{3,4}$ Longitudinal polarization at the interaction points is provided by spin rotators based on helical dipoles with large field integrals (with "strong" fields). It is not possible to control the deuteron polarization in such a collider.

In a figure- 8 collider, the spin first rotates about the vertical field in one arc. This rotation is then undone by the opposite field in the other arc. The resulting effect of the "strong" arc dipoles on the spin dynamics reduces to zero over one particle turn and the whole ring becomes "transparent" for the spin. Any spin orientation at any orbital location repeats every turn. In other words, in a figure- 8 accelerator, the spin tune is zero, and there is no preferred polarization orientation because the particle is in the zero-integer spin resonance region. To stabilize the beam polarization direction at the interaction point, it is sufficient to use compact insertions for polarization control, which utilize already existing collider magnets and solenoids with small field integrals ("weak" solenoids). Weak solenoids do not affect the closed orbit at all and essentially do not change the beam orbital parameters. This property is universal and does not depend on the particle type. Figure- 8 colliders provide a real opportunity for obtaining intense polarized deuteron beams with energies greater than a few tens of GeV.

Let us demonstrate how figure- 8 features help provide preservation and manipulation of the polarization in the ion complex of MEIC during experimental running.

\section{Acceleration and Spin Matching in Figure-8 Rings}

Figure 1 shows the schemes for polarization preservation in the pre-booster and large booster of MEIC. ${ }^{5}$ A weak solenoid stabilizes the polarization in the longitudinal direction in the straight where it is placed. Beam injection and extraction occur in the same straight of the accelerator. There is no problem with ramping up the fields of such solenoids during the acceleration cycle. The required solenoid field integral does not exceed $2 \mathrm{~T} \cdot \mathrm{m}$ at the top energy of the large booster for both protons and deuterons.

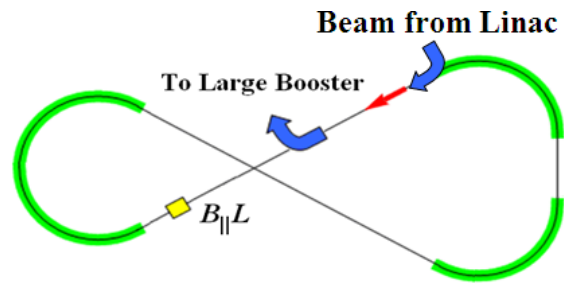

(a)

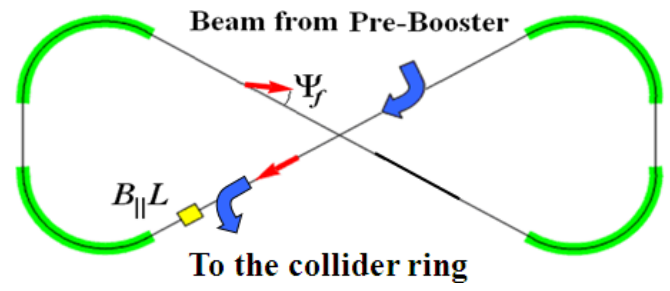

(b)

Fig. 1. Acceleration and spin matching in the pre-booster (a) and large booster (b). 


\section{Beam Polarization Manipulation in Figure-8 Colliders}

The advantages of figure- 8 colliders become especially prominent when there is a need to manipulate the polarization at the interaction point during an experiment. The unique capability to control the polarization with weak solenoids allows one to manipulate the particle spin at the interaction point with a negligibly small effect on the orbital motion. This greatly enhances the precision level of polarized beam experiments. By contrast, to manipulate the polarization during an experiment in a collider with two Siberian snakes, one uses a pair of spin rotators with the first one rotating the spin to the necessary polarization orientation at the interaction point and the second one returning the spin to its original orientation. ${ }^{3}$ Such spin rotators must use "strong fields", which affect not only the spin direction but also the orbital motion. It may result in adverse effects on the beam orbital properties such as betatron tune shift, changes of the dispersion and beta functions, etc., which must be taken into account during the experiment.

The beam polarization of any particles $\left(\mathrm{p}, \mathrm{d}, \mathrm{He}^{3}, \ldots\right)$ is controlled in MEIC using universal $3 \mathrm{D}$ spin rotators. ${ }^{5}$ The $3 \mathrm{D}$ rotators are designed using weak solenoids and allow performance of the following tasks: matching of the polarization direction at injection, polarization preservation during acceleration and storage, measurement of the beam polarization at any orbital location, and spin manipulation at the interaction point during experimental running.

Figure 2 shows an example of a 3D spin rotator for ion polarization control located at the entrance into an experimental straight. The rotator consists of three modules: those for control of the radial $n_{x}$, vertical $n_{y}$, and longitudinal $n_{z}$ components of the polarization (see Fig. 2a). The 3D spin rotator placement in the MEIC ring is shown schematically in Fig. $2 \mathrm{~b}$.

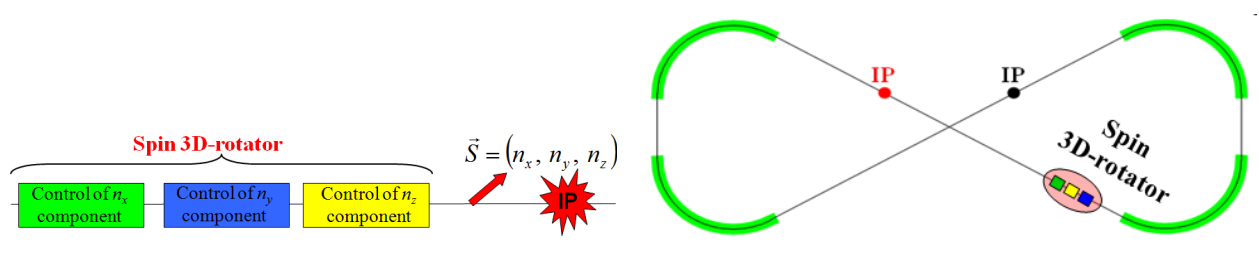

(a)

(b)

Fig. 2. Block diagram of a universal 3D spin rotator. (b) Spin rotator placement in the ion collider ring.

Figure 3a shows the module for control of the radial polarization component $n_{x}$, which consists of two pairs of opposite-field solenoids and three vertical-field dipoles producing a fixed orbit bump. The control module for the vertical polarization component $n_{y}$ is the same as that for the radial component except that the vertical-field dipoles are replaced with radial-field ones (Fig. 3b). To keep the orbit bumps fixed, the fields of the vertical- and radial-field dipoles must be ramped proportionally to the beam momentum. The module for control of the longitudinal polarization 


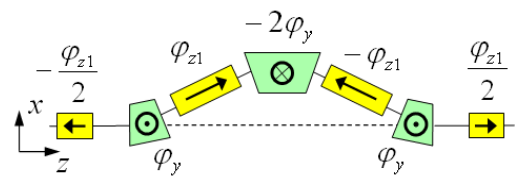

(a)

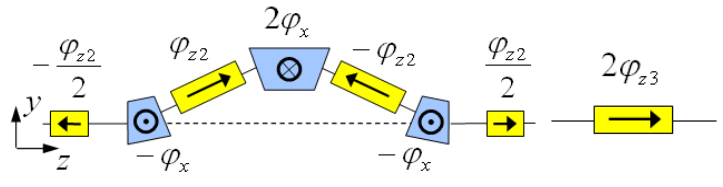

(b)

(c)

Fig. 3. Modules for control of the radial (a), vertical (b), and longitudinal (c) polarization components.

component $n_{z}$ consists of a single weak solenoid (Fig. 3c). There is a substantial flexibility in the placement and arrangement of these modules in the collider. For instance, to free up the space in the experimental straight, the module for control of the vertical polarization component can be installed anywhere in the arc.

Under the approximation of a small spin tune, the required spin rotation angles of the solenoids $\varphi_{z i}$ are determined by the following equations $(|\vec{n}|=1)$ :

$$
\varphi_{z 1}=\pi \nu \frac{n_{x}}{\sin \varphi_{y}}, \quad \varphi_{z 2}=\pi \nu \frac{n_{y}}{\sin \varphi_{x}}, \quad \varphi_{z 3}=\pi \nu n_{z}
$$

where $n_{x}, n_{y}$, and $n_{z}$ are the radial, vertical, and longitudinal polarization components, respectively, at the rotator's exit, $\varphi_{x}=G \gamma \alpha_{x}$ and $\varphi_{y}=G \gamma \alpha_{y}$ are the spin rotation angles of the aforementioned radial- and vertical-field dipoles, respectively, and $\alpha_{x}$ and $\alpha_{y}$ are the respective orbit bending angles of these dipoles. The calculation assumes that, with the solenoids off, the spin tune in the collider is zero.

\section{Calculation of the Beam Polarization in MEIC}

Let us present calculations of the proton and deuteron beam polarization in the MEIC collider with a single 3D rotator determining the equilibrium polarization at the interaction point. Schematic placement of the $3 \mathrm{D}$ rotator elements in the lattice of MEIC's experimental straight is shown in Fig. 4. The lattice's structural quadrupoles are shown in black, the vertical-field dipoles are green, the radial-field dipoles are blue, and the control solenoids are yellow. With each module's length of $\sim 5 \mathrm{~m}\left(L_{x}=L_{y}=0.5 \mathrm{~m}, L_{z}=1.0 \mathrm{~m}\right)$, the fixed orbit deviation in the bumps is $\sim 1.8 \mathrm{~cm}$ in the whole beam momentum range of the collider.

When using solenoids with the maximum solenoid field of $4 \mathrm{~T}$ in the $3 \mathrm{D}$ spin rotator, the proton and deuteron spin tunes are $\nu_{p}=0.01$ and $\nu_{d}=2.5 \cdot 10^{-4}$,

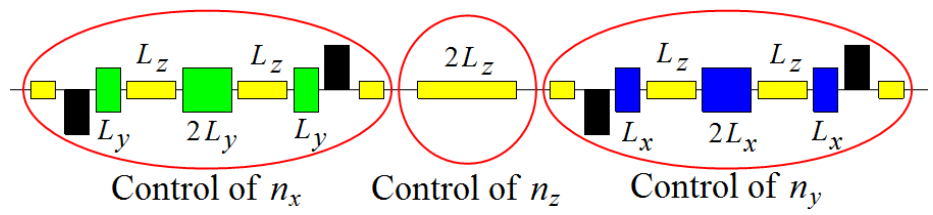

Fig. 4. Schematic placement of the 3D spin rotator elements. 


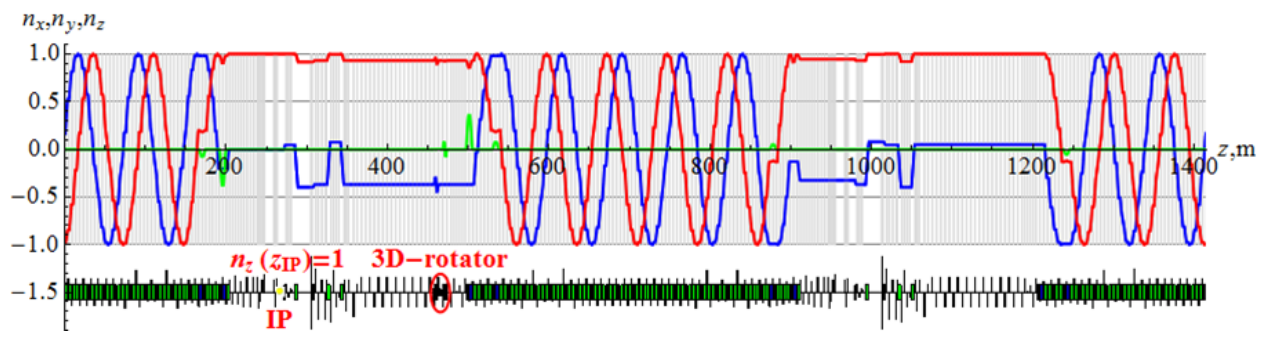

Fig. 5. Deuteron polarization in the ion collider ring for the longitudinal polarization configuration at the IP.

respectively. The magnetic fields of such solenoids can be changed sufficiently quickly on the time scale of several seconds that allows using the $3 \mathrm{D}$ rotator as a spinflipping system.

As an example, in Fig. 5, for an ideal collider structure, the equilibrium polarization components of a $100 \mathrm{GeV} / \mathrm{c}$ deuteron beam are shown as functions of the orbital length $z$ around the ring for the cases of longitudinal $\left(n_{z}\left(z_{I P}\right)=1\right)$ polarizations at the interaction point. The blue, red, and green curves show the radial, longitudinal, and vertical polarization components, respectively.

Note that the vertical polarization component is zero the whole ring with the exception of sections with radial fields. The polarization component lying in the ring's plane does not change significantly in the experimental straights and undergoes about 5 turns about the vertical axis in each arc. Because of this, the polarization has the same direction in both straights at $100 \mathrm{GeV} / \mathrm{c}$.

In Fig. 6, for an ideal collider structure, the equilibrium polarization components of a $100 \mathrm{GeV} / \mathrm{c}$ proton beam are shown as functions of the orbital length $z$ along the experimental straight for the cases of longitudinal polarizations at the interaction point. In contrast to the deuteron beam, the radial and longitudinal components of the proton polarization change significantly at each bending magnet of the lattice. The horizontal polarization component undergoes about 127 turns in each arc and is rotated significantly by the vertical-field dipoles located near the interaction point. The vertical polarization component, as in the deuteron case, remains unchanged in both experimental straights.

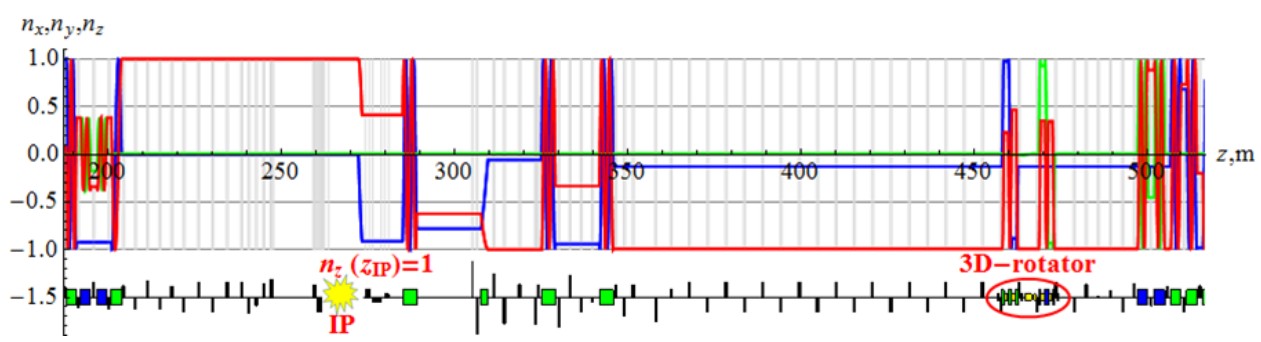

Fig. 6. Proton beam's polarization in the experimental straight of the MEIC ion collider ring. 


\section{Compensation of the Zero-Integer Spin Resonance Strength}

For polarization stability, the shift of the spin tune from zero provided by the $3 \mathrm{D}$ spin rotator in the collider must significantly exceed the strength of the zerointeger spin resonance: $\nu \gg w_{0}$. The strength of the zero-integer spin resonance consists of coherent and incoherent parts. The resonance strength is dominated by the coherent part, which is determined by lattice imperfections. The incoherent part of the resonance strength is determined by the beam emittances. The same type of $3 \mathrm{D}$ rotator as described above can be used to compensate the coherent part of the zero-integer resonance strength, which substantially reduces the required field integrals of the weak solenoids used in the 3D rotators. In this case, a real collider with polarized beams becomes equivalent to an "ideal" collider, which has its magnetic elements aligned exactly on the reference orbit so that the zero-integer resonance strength is determined only by the beam emittances. This allows for polarized beam experiments at a high precision level.

\section{Conclusions}

Let us briefly summarize the main features of the figure- 8 design, which allows for

- use of weak solenoids for polarization control at high energies of any particle type including deuterons,

- seamless integration of the spin control elements into the collider lattice with fixed closed orbit and no optics distortion,

- elimination of the resonant depolarization at all stages of the beam acceleration from the linac to the collider ring,

- adjustment of any polarization orientation at any orbital location (e.g., for spin matching at injection into the different accelerator complex components, polarimetry, spin flipping),

- manipulation of the particle spin during an experiment without affecting the beam orbital properties, which provides a capability of carrying out polarized beam experiments at a new precision level,

- compensation of the manufacturing and alignment errors of the lattice magnetic elements, which additionally substantially enhances the precision of polarized beam experiments, and

- ease of adjusting the spin dynamics to meet any experimental requirements, which may arise in the future.

\section{Acknowledgments}

This manuscript has been authored by Jefferson Science Associates, LLC under U.S. DOE Contracts No. DE-AC05-06OR23177 and DE-AC02-06CH11357. The U.S. Government retains a non-exclusive, paid-up, irrevocable, world-wide license to publish or reproduce this manuscript for U.S. Government purposes. 


\section{References}

1. Ya. S. Derbenev, University of Michigan report UM HE 96-05 (1996).

2. S. Abeyratne et al., "Science requirements and conceptual design for a polarized medium energy electron-ion collider at Jefferson lab", eds. Y. Zhang and J. Bisognano, arXiv: 1209.0757 [physics.acc-ph] (2012).

3. V. Ptitsin and Y. Shatunov, NIM A 398, 126 (1997).

4. E. Willen et al., in Proc. PAC99, NY, 3161 (1999).

5. A. M. Kondratenko et al., to appear in Proc. EIC'14. 